Review

\title{
Growth and Characterization on PMN-PT-Based Single Crystals
}

\section{Jian Tian * and Pengdi Han}

H. C. Materials Corporation, 479 Quadrangle Dr., Suite-E, Bolingbrook, IL 60440, USA;

E-Mail: han@hcmat.com

* Author to whom correspondence should be addressed; E-Mail: jtian@hcmat.com;

Tel.: +1-630-754-8623; Fax: +1-630-226-9010.

Received: 1 May 2014; in revised form: 2 July 2014 / Accepted: 3 July 2014 /

Published: 16 July 2014

\begin{abstract}
Lead magnesium niobate-lead titanate (PMN-PT) single crystals have been successfully commercialized in medical ultrasound imaging. The superior properties of PMN-PT crystals over the legacy piezoelectric ceramics lead zirconate titanate (PZT) enabled ultrasound transducers with enhanced imaging (broad bandwidth and improved sensitivity). To obtain high quality and relatively low cost single crystals for commercial production, PMN-PT single crystals were grown with modified Bridgman method, by which crystals were grown directly from stoichiometric melt without flux. For ultrasound imaging application, [001] crystal growth is essential to provide uniform composition and property within a crystal plate, which is critical for transducer performance. In addition, improvement in crystal growth technique is under development with the goals of improving the composition homogeneity along crystal growth direction and reducing unit cost of crystals. In recent years, PIN-PMN-PT single crystals have been developed with higher de-poling temperature and coercive field to provide improved thermal and electrical stability for transducer application.
\end{abstract}

Keywords: PMN-PT; PIN-PMN-PT; piezoelectric crystal; crystal growth; piezoelectric property 


\section{Introduction}

Lead magnesium niobate-lead titanate (PMN-PT) single crystals have superior dielectric and piezoelectric properties to the legacy lead zirconate titanate (PZT) polycrystalline ceramics, with electromechanical coupling factor $k_{33} \sim 0.9$, and piezoelectric charge coefficient $d_{33}>1500 \mathrm{pC} / \mathrm{N}$ [1-5]. During the past decade, significant progresses have been made on crystal growth and property characterization [6-9]. The Bridgman melt-growth method proven to be suitable for the growth of high quality large crystals [10]. For example, up to four-inch diameter PMN-PT crystals are now grown routinely with the [001] seeding. More recently progresses have been made in the fabrication of PMN-PT nanowires and nanowire-based nanocomposite $[11,12]$.

Lead indium niobate-lead magnesium niobate-lead titanate (PIN-PMN-PT) ternary crystals were developed to have improved thermal and electrical properties, as well as the excellent dielectric and piezoelectric properties similar to PMN-PT binary crystals [13-16]. Like PMN-PT, ternary PIN-PMN-PT crystals were successfully grown with modified Bridgman method along different crystallographic directions including the commonly used [001] and [011] directions.

For both PMN-PT and PIN-PMN-PT crystals grown by the melt method, the PT content gradually increases as crystal grows because of chemical segregation [8,17,18]. Crystal properties are closely related to the composition $[5,19]$, thus, variations in piezoelectric properties are expected along the crystal growth direction [20].

In this paper, crystal growth and property characterization of PMN-PT and PIN-PMN-PT are presented. Both Bridgman crystal growth and continuous feeding crystal growth are discussed. Selected properties along the growth direction of PMN-PT crystals grown by Bridgman method were characterized for the whole as-grown crystal boule to provide the full range of property variation. The piezoelectric charge coefficient $d_{33}$ and the free dielectric constant $K_{33}^{T}$ can increase by over $50 \%$ and $80 \%$, respectively, as the PT content increases from $27 \%$ to $32 \%$. In contrast, the electromechanical coupling factor $k_{33}$ showed a much smaller increase from 0.87 to 0.92 for the same PT range. Property improvements of the ternary PIN-PMN-PT crystals over the binary PMN-PT are also presented and discussed.

\section{Results and Discussion}

PMN-PT and PIN-PMN-PT single crystals were successfully grown with modified Bridgman method in both [001] and [011] directions. Selected dielectric and piezoelectric properties were measured along the growth direction of crystal boule to characterize the full property range in a crystal boule. Both PMN-PT and PIN-PMN-PT crystals show similar property variations along the growth direction as a result of compositional segregation during crystal growth.

\subsection{Crystal Growth}

Both PMN-PT and PIN-PMN-PT crystals grow directly from melt with Bridgman crystal growth method. High purity chemicals are used with no addition of other component like growth flux. As a result, the quality of as-grown crystal is generally superior to that grown with other techniques (e.g., flux growth). Because there is no dilution effect of the growth flux, crystal can grow at a faster rate 
without sacrificing the quality of the as-grown crystals. Faster growth rate in production means less growth time and reduced unit cost of the crystals. This is important for the commercialization of crystals where value for the price can be critical.

Both crystals can grow in different crystallographic directions, including [001], [011], [111], or [112]. Crystal growth along some direction (e.g., [001]) can be more challenging than in other directions (e.g., [011]). The growth along the [001] direction is the most challenging among all of the aforementioned directions for both PMN-PT and PIN-PMN-PT single crystals. This is also in line with the greater atomic distance in crystal lattice along [001] direction.

For medical ultrasound imaging application, there are two basic requirements for piezoelectric materials: (1) excellent $d_{33}$ mode properties; and (2) good property uniformity within a wafer. Because (001) wafers have the best $d_{33}$ mode property, (001) wafers are needed for ultrasound imaging. In addition, crystal growth along [001] is preferred to provide the best in-plane property uniformity as composition in a (001) wafer from a [001] grown crystal has the best uniformity.

Other application may favor crystals grown in a different direction. For example, some sonar application uses $d_{32}$ transverse mode. Because (011) wafers have the best $d_{32}$ mode properties, it makes sense to grow crystals in the [011] direction to get crystal parts with the best composition and, thus, the property uniformity within a crystal part.

Property variation across a wafer can be improved by selecting the appropriate growth direction. However, the compositional variation along the growth direction remains the challenges for Bridgman crystal growth. For Bridgman growth, all of the precursors melt before crystal growth starts. During crystal growth, the as-grown crystal has less PT content than the melt because of compositional segregation. PT concentration gradually increased in the remaining melt for both PMN-PT and PIN-PMN-PT crystals. As a result, the crystal grown at earlier stage has less PT compared to crystal grown later. This can lead to compositional and thus property variations within a part along the growth direction, especially for thick crystal parts. In addition, such compositional variation can pose challenge for crystal applications where tight property distribution is required within the group of crystal parts.

Continuous feeding crystal growth is designed to reduce the compositional and property variations along the growth direction. New materials are added into the platinum crucible during crystal growth process to keep the overall melt composition unchanged and thus the as-grown crystal composition constant. Because as-grown crystal has less PT concentration than in the melt due to compositional segregation, the newly added materials will have composition of less PT than the melt. Ideally the newly added materials have the same composition as the targeted as-grown crystals. The rate of new material addition equals the rate of crystal growth and the melt inside the platinum crucible maintains the constant composition and quantity.

Despite the elegant concept and great potential, there are challenges for continuous feeding crystal growth for PMN-PT and PIN-PMN-PT. To enable the feeding during crystal growth, the system has to be open at least during the material feeding. The lead and indium are both volatile at high temperatures. Extra precautions are needed to avoid the adverse environmental and health effects. The evaporated lead and indium oxides condensate on the wall of the feeding duct work, which can cause blockage or interruption of the feeding. Furthermore, the newly fed materials typically are at a lower temperature than the melt. The amount and temperature of feeding materials into the melt need to be controlled so that crystal growth process is not disturbed. 


\subsection{Property Variation of $P M N-P T$}

The PT content of melt-grown crystals increases along the growth direction due to chemical segregation [20]. Accordingly, the ferroelectric-paraelectric transition temperature $\left(T_{\mathrm{c}}\right)$ increases with increasing PT content [19]. Thus $T_{\mathrm{c}}$ can be used as an identifier for the composition of PMN-PT specimens [21,22]. In this paper, $T_{\mathrm{c}}$ is reported as the temperature for maximum $K^{T}$.

Specimens were selected along the growth direction. Figure 1 illustrates the dependence of $K^{T}$ (4400-7000) at room temperature on $T_{\mathrm{c}}\left(121-142^{\circ} \mathrm{C}\right)$. Specimen 0 was towards the base of the crystal at the early-stages of growth. The subsequent specimens, 1 through 16 , cover the majority compositional range used in piezoelectric applications. The $T_{\mathrm{c}}$ of $121^{\circ} \mathrm{C}$ to $142{ }^{\circ} \mathrm{C}$ corresponded to a PT variation of $26.5 \%-32 \%$ PT [8]. $K^{T}$ values up to 7500 or greater can be measured on samples with higher PT content closer to MPB (morphotropic phase boundary).

Figure 1. Dielectric property of crystals along growth direction.

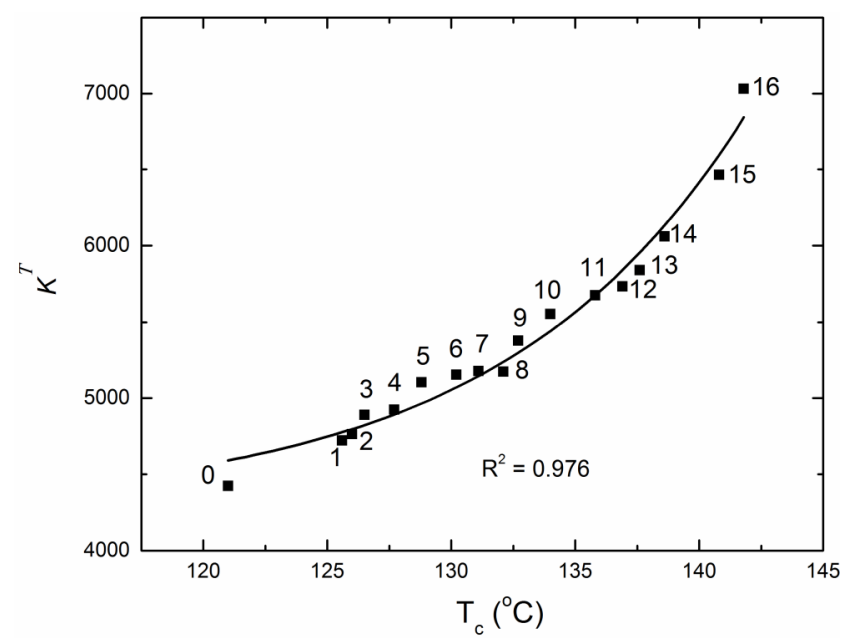

$k_{33}$ values obtained from selected crystal bars increase slightly with $T_{\mathrm{c}}$ (Figure 2 ). For example, $k_{33}$ increased from 0.84 to $0.92(9.5 \%)$ as $T_{\mathrm{c}}$ increased from $121{ }^{\circ} \mathrm{C}$ to $142{ }^{\circ} \mathrm{C}$ (i.e., $26.5 \%$ PT to $32 \% \mathrm{PT}$ ), with the majority of $k_{33}$ values greater than 0.87 , even though $K^{T}$ and $d_{33}$ increased significantly. In addition, $k_{33}$ appeared to approach an asymptotic value of $\sim 0.92$.

Figure 2. Coupling factor of crystals along growth direction.

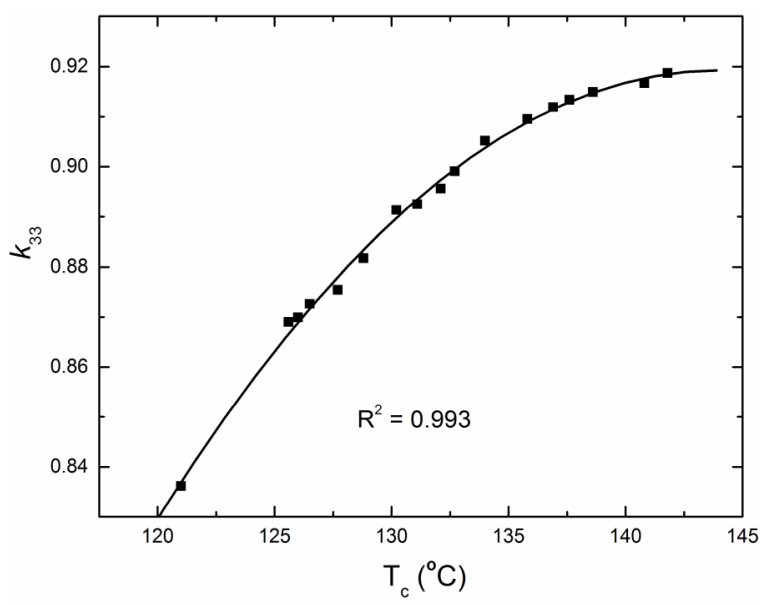


Constant electric field, $s_{33}^{E}$ values obtained for crystal bars by the resonance method increased significantly from $32.6 \times 10^{-12} \mathrm{~m}^{2} / \mathrm{N}$ to $62.6 \times 10^{-12} \mathrm{~m}^{2} / \mathrm{N}$, as $T_{\mathrm{c}}$ increased from $121{ }^{\circ} \mathrm{C}$ to $142{ }^{\circ} \mathrm{C}$ (i.e., $26.5 \% \mathrm{PT}$ to $32 \% \mathrm{PT}$ ). There is a strong linear dependence on $T_{\mathrm{c}}$ (and, thus, PT content) within the MPB region (Figure 3).

Figure 3. Compliance of crystals along growth direction.

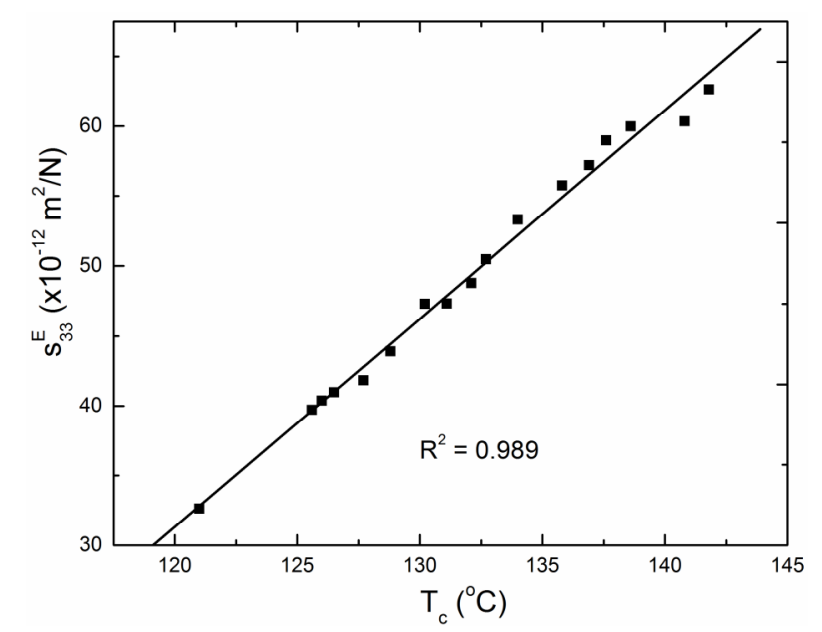

The $d_{33}$ values show a strong dependence on PT content (Figure 4). $d_{33}$ values increased from $950 \mathrm{pC} / \mathrm{N}$ to $1820 \mathrm{pC} / \mathrm{N}$, a increase of $\sim 90 \%$. There was a strong linear dependence of $d_{33}$ with $T_{\mathrm{c}}$, and, thus, PT content.

Figure 4. Piezoelectric property of crystals along growth direction.

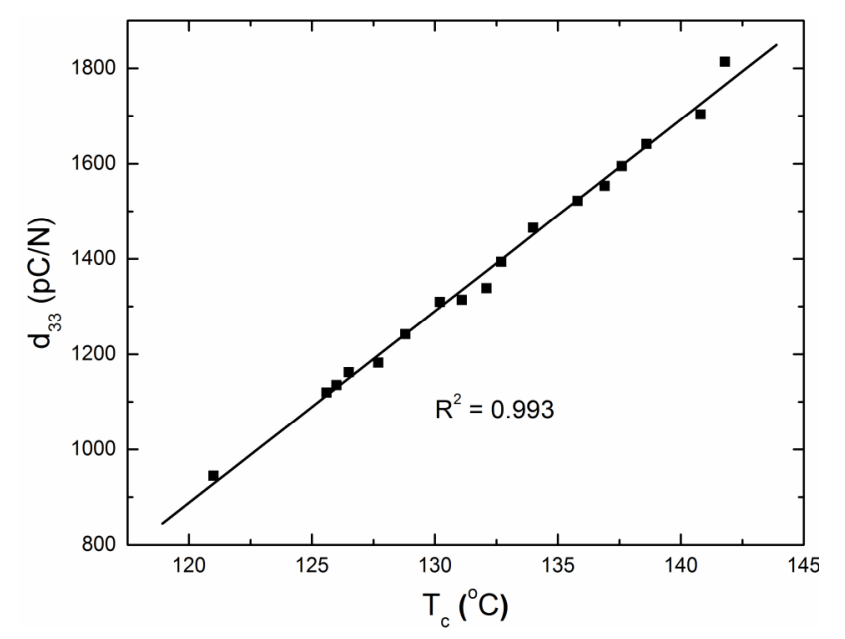

Property variations along the crystal growth direction have important implications for device applications. A most important fact is that the coupling factor $k_{33}$ remains relatively high and stable ( $k=0.87-0.92$ ) over a wide range of $d_{33}$ and $K^{T}$ values. The high coupling factors of PMN-PT single crystals enable transducer designs of high energy-conversion efficiency or broad bandwidth. The superior and variable $d_{33}$ and $K^{T}$ values allow a wide selection of piezoelectric and impedance parameters for design considerations.

As property variations occur along the growth direction, it is advantageous to choose crystals grown along preferred crystallographic directions for specific application. This is particularly true when 
in-plate homogeneity is critical especially for larger-sized crystal plates. More details have been presented in the crystal growth section.

\subsection{Property of PIN-PMN-PT}

Ternary PIN-PMN-PT single crystals are more complex than the binary PMN-PT crystals. In addition to the PT variation along the crystal growth direction similar to that of the PMN-PT crystals, PIN-PMN-PT crystals with different PIN contents can grow into piezoelectric single crystals. Fortunately, the PIN content does not vary much along the growth direction in a crystal boule. The variations in PIN content, as well as PT content, both can affect the property of the PIN-PMN-PT single crystals. Because the effect of PT content on the property of PIN-PMN-PT crystal is similar to that of the binary PMN-PT crystals, the focus of the section will be mainly on the effect of PIN content.

The main advantages of the ternary PIN-PMN-PT single crystals over the binary PMN-PT are the improved depoling temperature $\left(T_{\mathrm{R} / \mathrm{T}}\right)$ and the coercive field $\left(E_{\mathrm{C}}\right)$. The $T_{\mathrm{R} / \mathrm{T}}$ of PIN-PMN-PT crystals show an overall increasing trend with PIN content (Figure 5). The $T_{\mathrm{R} / \mathrm{T}} \mathrm{PIN} \%$ relation was fit well with an exponential growth curve (dotted line, $R^{2}>0.99$ ), which could be used to estimate the maximum $T_{\mathrm{R} / \mathrm{T}}$ of a PIN-PMN-PT crystal given the PIN content. PIN-PMN-PT crystals of high PIN content have further improved is $T_{\mathrm{R} / \mathrm{T}}$. The maximum $T_{\mathrm{R} / \mathrm{T}}$ for $0.33 \mathrm{PIN}-\mathrm{PMN}-\mathrm{PT}$ crystal is $137{ }^{\circ} \mathrm{C}$ [23], and $117^{\circ} \mathrm{C}$ for 0.24PIN-PMN-PT, compared to the $\max T_{\mathrm{R} / \mathrm{T}}$ of $97^{\circ} \mathrm{C}$ for PMN-PT crystal [14].

Figure 5. Correlation of $\max T_{\mathrm{R} / \mathrm{T}}$ and PIN\%.

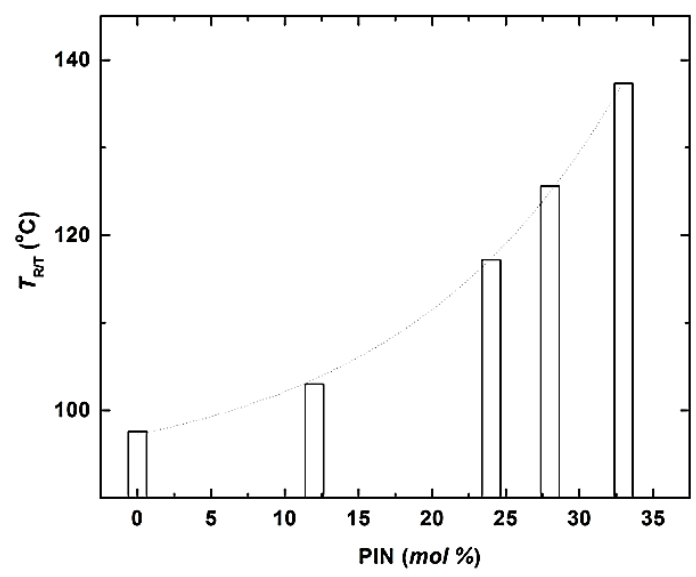

For piezocrystal application, $T_{\mathrm{R} / \mathrm{T}}$ instead of the Curie temperature $T_{\mathrm{c}}$, is the temperature limit. Crystal properties degrade rapidly when temperature is above $T_{\mathrm{R} / \mathrm{T}}$ for both PMN-PT and PIN-PMN-PT crystals. A higher $T_{\mathrm{R} / \mathrm{T}}$ enhances the transducer reliability by improving resistance to thermal de-poling, as well as allowing crystal application at higher temperatures. In addition, temperature-dependent property variation can be noticeably reduced with increased $T_{\mathrm{R} / \mathrm{T}}$. For example, the 0.33PIN-PMN-PT crystal has changes in the normalized $K^{T}$ with temperature similar to PZT-5H ceramic, superior to PMN-PT [8].

Since $E_{\mathrm{C}}$ values are relatively stable within a PIN-PMN-PT crystal boule, the average value of $E_{\mathrm{C}}$ is plot against the PIN content (Figure 6). The error bars show the standard deviation of the $E_{\mathrm{C}}$ from each crystal boule. The average $E_{\mathrm{C}}$ of crystals increased with PIN content. $E_{\mathrm{C}}$ exhibited a good linear 
correlation with PIN mol\% (dotted line, $R^{2}>0.99$ ). Compared to $T_{\mathrm{R} / \mathrm{T}}$, the magnitude of increase in $E_{\mathrm{C}}$ with PIN\% is less significant when PIN increased from 24 to 33 mol\%. Higher $E_{\mathrm{C}}$ allows PIN-PMN-PT crystals to be driven at a greater electric field with a reduced or zero bias field, which improves robustness of transducers based on PIN-PMN-PT crystals. $E_{\mathrm{C}}$ is relatively stable within a crystal boule for PIN-PMN-PT crystals of different PIN mol\%. The moderately steady PIN content likely led to the stable coercivity for PIN-PMN-PT crystals [24]. In contrast, variations in PMN and PT contents had little effect on crystal $E_{\mathrm{C}}$.

Figure 6. Correlation of average $E_{\mathrm{C}}$ and PIN\%.

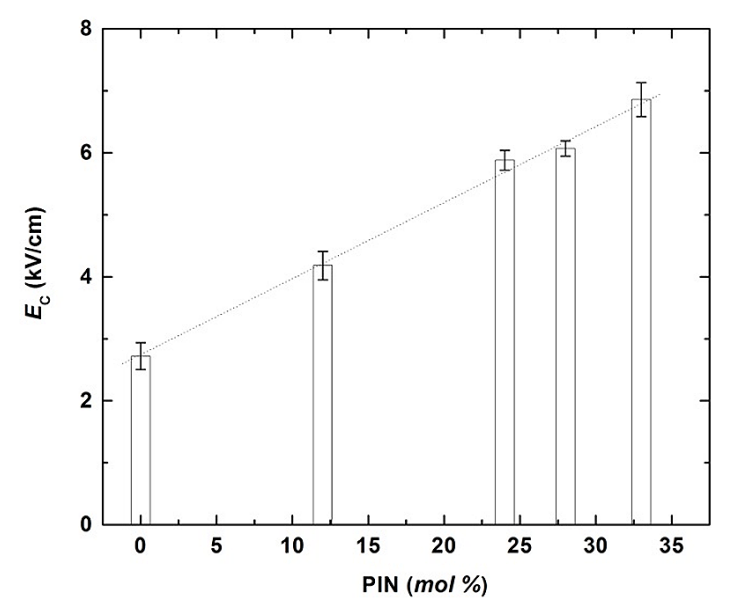

The improvements in $T_{\mathrm{R} / \mathrm{T}}$ and $E_{\mathrm{C}}$ of PIN-PMN-PT crystals expand the operating domains of crystals and enhance reliability for crystal transducers. Other piezoelectric properties, such as electromechanical coupling factors $k_{33}$ and $k_{\mathrm{t}}$ and piezoelectric strain coefficient $d_{33}$, remained excellent. The dielectric constant values for PIN-PMN-PT crystals decrease with increasing PIN mol\%.

\section{Experimental Section}

\subsection{Crystal Growth}

PMN-PT crystals are grown with modified Bridgman method along [001] and [011]. The starting composition is $31 \% \mathrm{PT}$, which is near the MPB in the rhombohedral phase for optimal dielectric and piezoelectric properties. Similar strategy was used when selecting the starting composition for crystal grow of the ternary PIN-PMN-PT single crystals. Crystal growth along [001] results in the best compositional homogeneity within the (001) wafers, while [011] growth for optimal compositional uniformity in the (011) wafers.

For Bridgman crystal growth, a small PMN-PT crystal seed (e.g., [001] or [011] orientation) is first loaded in the seed pocket at the bottom of the platinum crucible. Precursors with the appropriate composition are loaded in the platinum crucible. Crucible top is then sealed with a platinum cover. The platinum crucible loaded with crystal seed and precursor is placed into a crucible assembly that provides support to the platinum crucible when charge is melted at high temperature. The crucible assembly is then inserted into crystal growth furnace, where crystal grows for the next couple of weeks. One growth furnace can grow multiple crystals at the same time for improved efficiently. 
Precursor in the platinum crucible melts at temperatures greater than $1320^{\circ} \mathrm{C}$. Crystal seed at the bottom of the crucible is partially melted to form a solid/melt interface. When crucible assembly gradually lowered relative to the position of the furnace and temperature decreased, crystal growth starts from the solid/melt interface. Crystal growth conditions and growth rate are controlled to produce high quality crystals.

To improve the composition and property uniformity along the crystal growth direction, continuous feeding crystal growth was experimented. The basic concept of continuous feeding crystal growth is to feed materials of appropriate composition into the platinum crucible during crystal growth. As a result, the melt composition is maintained nearly constant during crystal growth with the newly added materials to balance out the compositional change due to the composition segregation from crystal growth.

\subsection{Sample Preparation}

Samples were selected along the crystal growth direction within the general usable portion of a boule, typically from the beginning of the full diameter of a crystal boule to the upper portion of the boule before MPB. The distance between samples is $1 \mathrm{~mm}$. Two types of crystal specimens, crystal plates of $7 \times 7 \times 0.7 \mathrm{~mm}^{3}$, and bars of $7 \times 0.7 \times 0.7 \mathrm{~mm}^{3}$, were selected along the length of [001] grown crystal boules. All of the faces of crystal plates and bars were orientated within $0.5^{\circ}$ of the (001) planes. To obtain the optimal compositional homogeneity within a specimen, the large face of crystal plates, and the length direction of crystal bars, were chosen to be normal to the growth direction [001]. In addition, crystal plates and bars of each group were prepared from the same location of a wafer. Gold electrodes were sputter coated on appropriate surfaces. Plates were poled in the thickness direction $\left(k_{\mathrm{t}}\right.$ mode) and bars along the length direction ( $k_{33}$ mode). The poling was done at $\sim 2 \times E_{\mathrm{C}}$ in air, with poling current monitored to completeness.

\subsection{Property Characterization}

All of the properties were determined at room temperature, $24 \mathrm{~h}$ after poling.

\subsubsection{Dielectric Properties}

The dielectric constant $K$ of crystal plates was calculated from geometry and capacitance measurements [18] on a Agilent 4294A Impedance Analyzer (Agilent Technologies Ltd., Tokyo, Japan). The free $\left(K^{T}\right)$ or clamped $\left(K^{\mathrm{S}}\right)$ dielectric constants were calculated from capacitance measurements at $1 \mathrm{kHz}$ and $1.5 f_{\mathrm{a}}$ respectively, where $f_{\mathrm{a}}$ is the anti-resonance frequency of the crystal plate.

\subsubsection{Electromechanical Coupling}

The rod extensional electromechanical coupling factor $k_{33}$ (longitudinal excitation) was determined from the aforementioned resonance frequencies $f_{p}$ and $f_{s}[25]$ :

$$
k_{33}{ }^{2}=\frac{\pi}{2} \frac{f_{s}}{f_{p}} \tan \left(\frac{\pi}{2} \frac{f_{p}-f_{s}}{f_{p}}\right)
$$




\subsubsection{Elastic Property}

Elastic constant was calculated from resonance measurements. The frequencies for maximum resistance $f_{p}$ and maximum conductance $f_{s}$ of crystal bars were determined on the impedance analyzer. From these characteristic frequencies, the compliances at constant electric field, $s_{33}^{E}$, were calculated, according to [26,27]:

$$
s_{33}^{E}=\frac{1}{4 \rho f_{p}^{2} l^{2}} \frac{1}{1-k_{33}{ }^{2}}=s_{33}^{D} \frac{1}{1-k_{33}{ }^{2}}
$$

where $\rho$ is the crystal density $\left(8.2 \mathrm{~g} / \mathrm{cm}^{3}\right), l$ the length of the bar $(7 \mathrm{~mm})$, and $k_{33}$ the electromechanical coupling factors for the longitudinal vibrations.

\subsubsection{Piezoelectric Properties}

Stable $d_{33}$ values for crystal bars could not be obtained from the $d_{33}$ meter due to the very small cross sectional area of the bars. Instead, $d_{33}$ values were calculated from other parameters including $k_{33}, s_{33}^{E}$ and $K^{T}[26,27]$. Values of $k_{33}$, and $s_{33}^{E}$ are from crystal bars and $K_{33}^{T}$ from crystal plates.

$$
d_{33, \text { Calc }}=k_{33} \sqrt{s_{33}^{E}} \sqrt{K^{T}}
$$

\subsubsection{Depoling Temperature}

Depoling temperature $T_{\mathrm{R} / \mathrm{T}}$ in the study is defined as the temperature of the first maximum free dielectric constant $K^{T}$ when a poled crystal sample is heated up gradually (e.g., $15^{\circ} \mathrm{C} / \mathrm{h}$ ) from room temperature to above the Curie temperature. The $K^{T}$ increases as temperature increases towards $T_{\mathrm{R} / \mathrm{T}}$. When temperature is above the depoling temperature, $K^{T}$ drops sharply and the temperature $T_{\mathrm{R} / \mathrm{T}}$ can be identified easily.

\section{Conclusions}

PMN-PT single crystals have been successfully commercialized in medical ultrasound imaging. Modified Bridgman technique proves to be the suitable technique for the commercial production of PMN-PT based single crystals with high quality and yield. Improved crystal growth techniques are promising in improving composition and property uniformity along the crystal growth direction, as well as reducing unit cost of the crystal. PIN-PMN-PT single crystals with improved thermal and electrical properties are advantageous for applications where higher drive and improved thermal performance are needed.

\section{Acknowledgments}

The research was supported by ONR and HC-R\&D funds. 


\section{Author Contributions}

The research was carried out by Jian Tian and Pengdi Han. Jian Tian prepared the manuscript and Pengdi Han helped the revision.

\section{Conflicts of Interest}

The authors declare no conflict of interest.

\section{References}

1. Park, S.E.; Shrout, T.R. Characteristics of relaxor-based piezoelectric single crystals for ultrasonic transducers. IEEE Trans. Ultrason. Ferroelectr. 1997, 44, 1140-1147.

2. Luo, H.S.; Xu, G.S.; Wang, P.C.; Yin, Z.W. Growth and characterization of relaxor ferroelectric PMNT single crystals. Ferroelectrics 1999, 231, 685-690.

3. Fu, H.X.; Cohen, R.E. Polarization rotation mechanism for ultrahigh electromechanical response in single-crystal piezoelectrics. Nature 2000, 403, 281-283.

4. Bokov, A.A.; Ye, Z.G. Ferroelectric properties of monoclinic $\mathrm{Pb}\left(\mathrm{Mg}_{1 / 3} \mathrm{Nb}_{2 / 3}\right) \mathrm{O}_{3}-\mathrm{PbTiO}_{3}$ crystals. Phys. Rev. B 2002, 66, 1-5.

5. Luo, H.S.; Xu, G.S.; Xu, H.Q.; Wang, P.C.; Yin, Z.W. Compositional homogeneity and electrical properties of lead magnesium niobate titanate single crystals grown by a modified Bridgman technique. Jpn. J. Appl. Phys. 2000, 39, 5581-5585.

6. Zhao, X.Y.; Fang, B.J.; Cao, H.; Guo, Y.P.; Luo, H.S. Dielectric and piezoelectric performance of PMN-PT single crystals with compositions around the MPB: Influence of composition, poling field and crystal orientation. Mater. Sci. Eng. B Solid 2002, 96, 254-262.

7. Lee, S.H.; Lee, S.S.; Roh, Y.; Lee, H.Y.; Han, J.H. Measurement of all the elastic, piezoelectric, and dielectric properties of PMN-PT single crystals. Proc. SPIE 2003, 5053, 505-512.

8. Tian, J.; Han, P.; Payne, D.A. Measurements along the growth direction of PMN-PT crystals: Dielectric, piezoelectric, and elastic properties. IEEE Trans. Ultrason. Ferroelectr. 2007, 54, 1895-1902.

9. Zhang, S.; Li, F. High performance ferroelectric relaxor- $\mathrm{PbTiO}_{3}$ single crystals: Status and perspective. J. Appl. Phys. 2012, 111, doi:10.1063/1.3679521.

10. Han, P.; Tian, J.; Yan, W. Handbook of Advanced Dielectric, Piezoelectric and Ferroelectric Materials: Synthesis, Properties and Applications; Woodhead Publishing Ltd.: Cambridge, England, 2008.

11. Xu, S.; Poirier, G.; Yao, N. PMN-PT nanowires with a very high piezoelectric constant. Nano Lett. 2012, 12, 2238-2242.

12. Xu, S.; Yeh, Y.-W.; Poirier, G.; McAlpine, M.C.; Register, R.A.; Yao, N. Flexible piezoelectric PMN-PT nanowire-based nanocomposite and device. Nano Lett. 2013, 13, 2393-2398.

13. Xu, G.; Chen, K.; Yang, D.F. Growth and electrical properties of large size $\mathrm{Pb}\left(\mathrm{In}_{1 / 2} \mathrm{Nb}_{1 / 2}\right) \mathrm{O}_{3^{-}}$ $\mathrm{Pb}\left(\mathrm{Mg}_{1 / 3} \mathrm{Nb}_{2 / 3}\right) \mathrm{O}_{3}$ crystals prepared by the vertical Bridgman technique. Appl. Phys. Lett. 2007, 90, doi:10.1063/1.2431706. 
14. Tian, J.; Han, P.; Huang, X.; Pan, H.; Carroll, J.F.; Payne, D.A. Improved stability for piezoelectric crystals grown in the lead indium niobate-lead magnesium niobate-lead titanate system. Appl. Phys. Lett. 2007, 91, doi:10.1063/1.2817743.

15. Luo, J.; Hackenberger, W.; Zhang, S.J.; Shrout, T.R. Elastic, piezoelectric and dielectric properties of PIN-PMN-PT crystals grown by Bridgman method. Proc. IEEE Ultrason. Symp. 2008, 1-4, 261-264.

16. Zhang, S.J.; Luo, J.; Hackenberger, W.; Sherlock, N.P.; Meyer, R.J.; Shrout, T.R. Electromechanical characterization of $\mathrm{Pb}\left(\mathrm{In}_{0.5} \mathrm{Nb}_{0.5}\right) \mathrm{O}_{3}-\mathrm{Pb}\left(\mathrm{Mg}_{1 / 3} \mathrm{Nb}_{2 / 3}\right) \mathrm{O}_{3}-\mathrm{PbTiO}_{3}$ crystals as a function of crystallographic orientation and temperature. J. Appl. Phys. 2009, 105, doi:10.1063/1.3131622.

17. Wang, P.-C.; Luo, H.-S.; Pan, X.-M.; Li, D.-L.; Yin, Z.-W. Dielectric and piezoelectric properties of PMN-PT single crystals grown by Bridgman method. Proc. IEEE Ultrason. Symp. 2000, 2 , 537-540.

18. Tian, J.; Han, P.; Carroll, J.F.; Payne, D.A. Growth and improved properties of ternary piezoelectric single crystals in lead indium niobate-lead magnesium niobate-lead titanate system. Proc. IEEE Ultrason. Symp. 2009, 972-975.

19. Zawilski, K.T.; Custodio, M.C.C.; DeMattei, R.C.; Lee, S.G.; Monteiro, R.G.; Odagawa, H.; Feigelson, R.S. Segregation during the vertical Bridgman growth of lead magnesium niobate-lead titanate single crystals. J. Cryst. Growth 2003, 258, 353-367.

20. Cao, H.; Fang, B.J.; Xu, H.Q.; Luo, H.S. Effects of segregation on composition and dielectric and piezoelectric properties of $\mathrm{Pb}\left(\mathrm{Mg}_{1 / 3} \mathrm{Nb}_{2 / 3}\right) \mathrm{O}_{3}-38 \% \mathrm{PbTiO}_{3}$ single crystal. J. Inorg. Mater. 2003, 18, $50-56$.

21. Shrout, T.R.; Chang, Z.P.; Kim, N.C.; Markgraf, S. Dielectric behavior of single-crystals near the $(1-x) \mathrm{Pb}\left(\mathrm{Mg}_{1 / 3} \mathrm{Nb}_{2 / 3}\right) \mathrm{O}_{3}-x \mathrm{PbTiO}_{3}$ morphotropic phase-boundary. Ferroelectr. Lett. 1990, 12, 63-69.

22. Zekria, D.; Shuvaeva, V.A.; Glazer, A.M. Birefringence imaging measurements on the phase diagram of $\mathrm{Pb}\left(\mathrm{Mg}_{1 / 3} \mathrm{Nb}_{2 / 3}\right) \mathrm{O}_{3}-\mathrm{PbTiO}_{3}$. J. Phys. Condens. Matter 2005, 17, 1593-1600.

23. Tian, J.; Han, P.; Carroll, J.F.; Payne, D.A. Improved Properties of Piezoelectric Crystal Lead Indium Niobate-Lead Magnesium Niobate-Lead Titanate. Proc. IEEE Ultrason. Symp. 2008, 1-4, 269-271.

24. Tian, J.; Han, P. Crystal growth and property characterization for PIN-PMN-PT ternary piezoelectric crystals. $J$. Adv. Dielectr. 2014, 4, doi:10.1142/S2010135X13500276.

25. Moulson, A.J.; Herbert, J.M. Electroceramics, 2nd ed.; Wiley: Hoboken, NJ, USA, 2003.

26. IEEE Standard on Piezoelectricity; The Institute of Electrical and Electronics Engineerings, Inc: New York, NY, USA, 1987.

27. Li, Y.; Qin, Z.; Zhou, Z. Measurement of Piezoelectric and Ferroelectric Materials; Science Press: Beijing, China, 1984. (In Chinese)

(C) 2014 by the authors; licensee MDPI, Basel, Switzerland. This article is an open access article distributed under the terms and conditions of the Creative Commons Attribution license (http://creativecommons.org/licenses/by/3.0/). 